\title{
Efecto de la inclusión de zeolita (clinoptilolita) en ovinos en etapa de finalización: Respuesta productiva y energética de la dieta
}

\author{
Estrada-Angulo, A. ' ; Coronel-Burgos, F. ' ; Castro Pérez, B.I. ${ }^{\text {; }}$ López Soto, M.A. ${ }^{2}$; Barreras, A. ${ }^{2}$; Angulo-Montoya, C. ; \\ Contreras Pérez, G.' y Plascencia, A. ${ }^{2 @}$
}

'Facultad de Medicina Veterinaria y Zootecnia. Universidad Autónoma de Sinaloa. México.

${ }^{2}$ Instituto de Investigaciones en Ciencias Veterinarias. Universidad Autónoma de Baja California. México.

\section{PALABRAS CLAVE ADICIONALES}

Borregos de pelo.

Zeolita.

Eficiencia energética.

Dieta de finalización.

\section{ADDITIONAL KEYWORDS}

Hairy sheep.

Zeolite.

Energy efficiency.

Finishing diet.

\section{INFORMATION}

\section{Cronología del artículo.}

Recibido/Received: 26.08 .2016

Aceptado/Accepted: 07.03.2017

On-line: 15.07.2017

Correspondencia a los autores/Contact e-mail:

alejandro.plascencia@uabc.edu.mx

\section{RESUMEN}

El objetivo de este estudio fue evaluar el efecto de la adición de diferentes niveles $(0,1,5,3$ y $4,5 \%$ ) de zeolita en dietas integrales de finalización sobre la respuesta productiva y la eficiencia en la utilización de la energía neta de la dieta de ovinos de pelo. Para lo anterior, 40 ovinos machos $3 / 4$ Katahdin $\times 1 / 4$ Pelibuey (peso inicial $=32,28 \pm 2,34 \mathrm{~kg}$ ) se asignaron a 20 corraletas ( 2 ovinos/corraleta, 5 corrales/tratamiento) alimentándose durante 75 días con alguno de los 4 tratamientos. Las dietas ofrecidas fueron tipo integral. La dieta testigo (sin zeolita) contenía $16,51 \%$ de proteína cruda (PC) y 1,39 McalENg/ $\mathrm{kg}$ y la cantidad total de zeolita incluida en cada tratamiento sustituyó al maíz y a la pasta de soja en partes iguales. Por cada nivel de sustitución del maíz-pasta de soja se disminuyó la concentración de PC en 0,1 1 puntos porcentuales y la energía neta en $0,03 \mathrm{Mcal} / \mathrm{kg}$. El sustituir maíz y pasta de soja por zeolita hasta un nivel de $1,5 \%$ en la dieta no afectó el consumo de materia seca (MS), la ganancia diaria, la eficiencia alimenticia, la utilización de la energía neta de la dieta (EN observada sobre la EN esperada) - la retención aparente de energía por unidad de MS consumida. El aumentar a el nivel de suplementación de zeolita por encima de 1,5\% incrementó la eficiencia alimenticia, la utilización de la energía neta de la dieta y la retención aparente de energía por unidad de MS consumida siendo la respuesta máxima para el nivel de inclusión de $3 \%$ (componente cuadrático, $p<0,01$ ). Con respecto al grupo testigo, la inclusión de $3 \%$ de zeolita a la dieta mejoró ( $p<0,01)$ en $6,8 \%$ la eficiencia alimenticia, en $8.4 \%$ la utilización de la energía neta de la dieta y en $8,9 \%$ la retención aparente de energía por unidad de MS consumida. La inclusión de zeolita por encima de 1,5\% a dieta de finalización mejora la utilización de la energía neta de la dieta. En base al rendimiento productivo y la eficiencia en la utilización de la energía neta de la dieta, la respuesta óptima observada para los corderos en el presente estudio fue con el $3 \%$ de inclusión de zeolita.

\section{Effect of zeolite inclusion for finishing feedlot lambs: Growth performance and dietary energetic}

\section{SUMMARY}

The aim of this study was to evaluate the effect of different supplemental levels $(0,1.5,3$ and $4.5 \%$ ) of zeolite in total mixed finishing diets on the productive response and efficiency of using dietary net energy in hairy lambs. For this aim, 40 3/4Katahdin $\times 1 / 4$ Pelibuey lambs (initial weight $=32.28 \pm 2.34 \mathrm{~kg}$ ) were assigned to 20 pens ( 2 lambs/pen, 5 pens/treatment) and were fed for 75 days on one of the four treatments. Control diet (no supplemental zeolite) contained $16.51 \% \mathrm{CP}$ and $1.39 \mathrm{McalNEg} / \mathrm{kg}$, and the total quantity of zeolite included replaced corn and soybean meal in equal parts. For each level of which corn and soybean meal were substituted, $\mathrm{CP}$ concentration decreased 0.11 percentage points and net energy decreased $0.03 \mathrm{McalNEg} / \mathrm{kg}$. The partial replacement of corn and soybean meal by zeolite at a level of $1.5 \%$ in the diet did not affect DM intake, daily gain, feed efficiency, dietary net energy (observed-to- expected $\operatorname{diet} \mathrm{NE}$ ) or the apparent energy retention per unit of dry matter intake (DMI). Increasing the level of zeolite supplementation above $1.5 \%$, increased feed efficiency, dietary net energy and apparent energy retention per unit of DMl averaging 4.5, 6.4 and $7.4 \%$; respectively. The maximun response was obtained for an inclusion level of $3 \%$ (quadratic component, $p<0.01$ ). Compared to controls, the inclusion of $3 \%$ of zeolite in the diet improved ( $p<0.01$ ) feed efficiency by $6.8 \%$, dietary net energy by $8.4 \%$, and apparent energy retention per unit of DMI by $8.9 \%$. The use of zeolite in finishing diets improves the utilization of dietary net energy. Based on growth performance and efficiency of utilization of dietary net energy, the optimal response observed for lambs in the present study was obtained for the level of $3 \%$ of zeolite inclusion. 


\section{INTRODUCCIÓN}

Las zeolitas naturales son una familia mineral de origen volcánico constituida por aluminosilicatos hidratados de estructura tetraédrica de forma tridimensional que se interconectan por compartir átomos de oxígeno, esta característica molecular genera la formación de canales y cavidades bien definidos (Mumpton y Fishman, 1977; Ostrooumov, 2002) con gran capacidad de intercambio de agua y cationes (Inglezakis y Zorpas, 2012). La clinoptilolita cálcica es la zeolita natural más abundante y debido a su gran capacidad de intercambio catiónico es ampliamente utilizada en la agricultura. Por sus características adsorbentes, que modifican la viscosidad de los fluidos (Spotti et al., 2005), y su gran afinidad para retener y liberar nitrógeno amoniacal $\left(\mathrm{N}-\mathrm{NH}_{3}\right)$, las zeolitas son prometedoras como aplicación en aditivos alimenticios para rumiantes (Mumpton y Fishman, 1977) ya que estos efectos pueden favorecer la eficiencia de la fermentación ruminal y la absorción de nutrimentos, reflejándose en una mayor eficiencia en la utilización de la energía neta aparente de la dieta. Sin embargo, los efectos sobre la utilización de nutrimentos y de respuesta productiva en rumiantes suplementados con zeolitas son inconsistentes ya que en varios estudios se ha informado de respuestas positivas (Pond, 1984; Ghaemnia et al., 2010; Goodarzi y Nanekarani, 2012), mientras que en otros, no se han observado ventajas, o incluso, se informan resultados negativos cuando las zeolitas son utilizadas (Galyean y Chabot, 1981; Sherwood et al., 2005). Las razones para la inconsistencia de los resultados pueden deberse, en parte, por el nivel de zeolita utilizado (McCollumy Galyean, 1983) y por la composición de la dieta empleada (Pond, 1989). La mayoría de los informes con resultados positivosson en dietas de moderadas a bajas en contenido de energía suplementadas con zeolita en un rango de 1 a $5 \%$ de la materia seca (Ghaemnia et al., 2010; Goodarzi y Nanekarani 2012; Ural, 2014); mientras que, la información sobre la respuesta al uso de zeolitas en rumiantes consumiendo dietas de finalización altas en energía es limitada. Por lo anterior, el objetivo de este estudio fue evaluar el efecto de la adición de diferentes niveles de una zeolita natural (clinoptilolita) sobre la respuesta productiva y la eficiencia en la utilización de la energía neta aparente de la dieta de ovinos en fase de finalización.

\section{MATERIALES Y MÉTODOS}

El experimento se llevó a cabo en la Unidad Experimental para Engorda de Pequeños Rumiantes ubicado en las instalaciones de la Facultad de Medicina Veterinaria y Zootecnia de la Universidad Autónoma de Sinaloa, ubicado en Culiacán, México (24 $46^{\prime} 13^{\prime \prime} \mathrm{N}$ and $107^{\circ} 21^{\prime}$ $\left.14^{\prime \prime} \mathrm{W}\right)$ situado a $55 \mathrm{~m}$ sobre el nivel del mar y con clima tropical.

Todos los procedimientos de manejo de animales se realizaron dentro de las pautas de técnicas aprobadas nacionalmente para el uso y cuidado de animales (NOM-051-ZOO-1995: para el cuidado humanitario de los animales durante la movilización de animales; NOM-062-ZOO-1999: especificaciones técnicas para el cuidado y uso de animales de laboratorio granjas de ganado, granjas, centros de producción, reproducción y cría, zoológicos y sala de exposiciones, deben cumplir con los principios básicos de bienestar de los animales; NOM-024-ZOO-1995: estipulaciones de salud animal y características zoosanitarias durante el transporte de animales).

Para valorar el efecto de la suplementación de zeolita sobre el comportamiento productivo y la eficiencia en la utilización de la energía neta aparente de la dieta en corderos alimentados con dietas de finalización se utilizaron 40 ovinos machos enteros cruzados de las razas $3 / 4$ Katahdin $\times 1 / 4$ Pelibuey (peso vivo promedio $=$ $32,28 \pm 2,34 \mathrm{~kg}$ ) en una prueba de rendimiento productivo con duración de 75 días. Cuatro semanas antes del inicio del experimento los corderos fueron tratados vía oral para endoy exoparásitos $(2,5 \mathrm{mg} / \mathrm{kg}$ PV, Closantel ${ }^{\circledR} \% \%$, Panavet de México), inyectados con $1 \times 10^{6}$ UI de vitamina A (Synth-AD®, Fort Dodge, de Sanidad Animal, México) y se iniciaron en la adaptación a la dieta de finalización (sin zeolita). Los corderos se pesaron al inicio del experimento (báscula electrónica; TORREY TIL/S: 107 2691, TORREY electronics Inc., Houston, TX, USA).El peso inicial se analizó colateralmente en el análisis estadístico de aplicación del programa SAS, resultando significativo $(\mathrm{p}<0.001)$, corroborando la necesidad de considerarlo en el modelo como fuente de variación (bloque) para evaluar limpiamente los efectos medios de los tratamientos como única hipótesis estadística a evaluar. De tal forma se conformaron 5 bloques (grupos) de peso distribuyéndose los 40 borregos en 20 corraletas ( 2 borregos por corraleta con 5 repeticiones por tratamiento) de $2 \times 3 \mathrm{~m}$ completamente sombreadas con bebedero automático y comedero en línea. Los tratamientos consistieron en adicionar a una dieta de finalización tipo integral (dieta totalmente mezclada en mezclador horizontal cap. 1400 kg, H.C. Davis SonsManufacturers, mod. MD-5, Bonner Springs, KS, USA) formulada en base a maíz quebrado (proporción forraje:concentrado de 9:91) cuatro niveles de zeolita (tabla I) sustituyendo parcialmente al maíz y a la pasta de soja de la siguiente manera: 1) Dieta testigo, sin zeolita, 2) 1,5\% de zeolita sustituyendo $0,75 \%$ de maíz y $0,75 \%$ de pasta de soja, 3) 3\% de zeolita sustituyendo $1,5 \%$ de maíz y 1,5\% de pasta de soja y 4) $4,5 \%$ de zeolita sustituyendo $2,25 \%$ de maíz y 2,25\% de pasta de soja. La fuente de zeolita utilizada fue clinoptilolita cálcica (ZEO-SIL; Grupo TCDN, Puebla, México). Previo a la incorporación a las dietas, la zeolita se mezcló con elementos menores (urea, roca caliza y sal mineralizada) para finalmente incorporarse como tercer paso de mezclado en la elaboración de las dietas experimentales que contuvieron zeolita. Los corderos tuvieron acceso libre a las dietas experimentales ajustándose el ofrecido para tener un mínimo de rechazo ( $<5 \%$ del total ofrecido) en comedero. El alimento se ofreció 2 veces al día (a las 0800 y 1400 h) en una proporción aproximada de 30:70 de lo consumido diariamente, 15 minutos previos al suministro matutino de alimento se revisaron visualmente los comederos, de existiralimento rechazado se recogió y se pesó y el consumo se registró diariamente. Los ajustes de consumo diario (incremento o disminución de la cantidad ofrecida) se realizaron en el alimento ofrecido de la tarde del día siguiente. A las dietas 
Tabla I. Ingredientes y composición química de las dietas integrales utilizadas (Ingredients and chemical composition of total mixed diets utilized).

\begin{tabular}{|c|c|c|c|c|}
\hline \multirow{2}{*}{ Concepto } & \multicolumn{4}{|c|}{ Nivel de zeolita, \% MS } \\
\hline & 0 & 1.5 & 3.0 & 4,5 \\
\hline \multicolumn{5}{|c|}{ Composición del ingrediente, \% MS } \\
\hline Maíz quebrado & 60,50 & 59,75 & 59,00 & 58,25 \\
\hline Pasta de soja & 16,00 & 15,25 & 14,50 & 13,75 \\
\hline Heno de Sudán molido & 9,00 & 9,00 & 9,00 & 9,00 \\
\hline Zeolita (Clinoptilolita) ${ }^{*}$ & 0,00 & 1,50 & 3,00 & 4,50 \\
\hline Grasa animal & 2,00 & 2,00 & 2,00 & 2,00 \\
\hline Sal mineralizada ${ }^{\dagger}$ & 2,50 & 2,50 & 2,50 & 2,50 \\
\hline Melaza de caña & 10,00 & 10,00 & 10,00 & 10,00 \\
\hline \multicolumn{5}{|c|}{ Concentración de energía, Mcal/kg de $\mathrm{MS}^{\ddagger}$} \\
\hline $\mathrm{EN}_{\mathrm{m}}, \mathrm{Mcal} / \mathrm{kg}$ & 2,04 & 2,01 & 1,98 & 1,95 \\
\hline $\mathrm{EN}_{\mathrm{g}}, \mathrm{Mcal} / \mathrm{kg}$ & 1,39 & 1,37 & 1,35 & 1,33 \\
\hline \multicolumn{5}{|c|}{ Composición de nutrientes, \% MS } \\
\hline Proteína cruda & 16,51 & 16,11 & 15,71 & 15,30 \\
\hline Extracto etéreo & 5,27 & 5,22 & 5,17 & 5,11 \\
\hline Fibra detergente neutro & 14,85 & 14,66 & 14,47 & 14,27 \\
\hline Cenizas & 6,88 & 8,42 & 9,96 & 11,49 \\
\hline
\end{tabular}

*Clinoptilolita cálcica (Zeo-Sil, Grupo TCDN, Puebla, Puebla); ${ }^{\dagger}$ contenido mineral de la sal mineralizada: $\mathrm{CoSO}_{4}, 0,068 \%$; $\mathrm{CuSO}_{4}, 1,04 \%$; $\mathrm{FeSO}_{4}, 3,57 \%$; $\mathrm{ZnO}, 1,24 \%$; $\mathrm{MnSO}_{4}, 1,07 \%$, KI 0,052\% y NaCl, 92,96\%; calculada a través de los valores tabulares expresados en el NRC (2007).

experimentales se les realizaron las siguientes determinaciones: materia seca (MS, secado al horno a $105^{\circ}$ C hasta peso constante; método 930.15; AOAC, 2000); proteína cruda (PC, $\mathrm{N} \times 6.25$, método 984.13; AOAC, 2000); cenizas (método 942.05; AOAC, 2000); fibra detergente neutro (FDN, Van Soest et al., 1991, corregido para cenizas) y extracto etéreo (método 920.39; AOAC, 2000).

Para efecto de la estimación del rendimiento productivo (ganancia y eficiencia alimenticia) y la estimación de la energía neta observada de la dieta, el peso vivo inicial se multiplicó por el coeficiente 0,96 para ajustar el relleno gastrointestinal (PVmermado, Cannas et al., 2004), y para el registro del peso final a todos los corderos se les retiró el alimento (el agua potable no fue retirada) durante $18 \mathrm{~h}$ antes de registrar el peso final. El promedio de la ganancia diaria de peso (GDP) se estimó como sigue: (peso inicial- peso final)/75. La eficiencia alimenticia se calculó dividiendo la GDP entre el promedio de consumo diario de MS. El consumo esperado de MS para cada uno de los tratamientos se estimó mediante las siguientes ecuaciones (NRC, 2007; Estrada et al., 2013):

ZEOL-0: Consumo MS esperado, $\mathrm{kg} / \mathrm{d}=(0,056$ $\left.\mathrm{PV}^{0.75} / 2,04\right)+\left(0,276 \times \mathrm{GDP} \times \mathrm{PV}^{0.75} / 1,39\right)$

ZEOL-1,5: Consumo MS esperado, $\mathrm{kg} / \mathrm{d}=$ $\left(0,056 \mathrm{PV}^{0.75} / 2,01\right)+\left(0,276 \times \mathrm{GDP} \times \mathrm{PV}^{0.75} / 1,37\right)$

ZEOL-3: Consumo MS esperado, $\mathrm{kg} / \mathrm{d}=(0,056$ $\left.\mathrm{PV}^{0.75} / 1,98\right)+\left(0,276 \times \mathrm{GDP} \times \mathrm{PV}^{0.75} / 1,35\right)$.

ZEOL-4,5: Consumo MS esperado, $\mathrm{kg} / \mathrm{d}=$ $\left(0,056 \mathrm{PV}^{0.75} / 1,95\right)+\left(0,276 \times \mathrm{GDP} \times \mathrm{PV}^{0.75} / 1,33\right)$.
Donde los valores expresados en el numerador corresponden a los requerimientos de energía para mantenimiento y ganancia, y los valores expresados en el denominador representan la concentración energética de cada una de las dietas (tabla I). El coeficiente $(0,276)$ se estimó para el peso adulto de $113 \mathrm{~kg}$ para corderos Kathadin $\times$ Pelibuey (NRC, 1985; Estrada-Angulo et al., 2013). La EN observada para las dietas se estimó por medio de la fórmula cuadrática:

$$
x=\left(-\mathrm{b}-\sqrt{ } \mathrm{b}^{2}-4 \mathrm{ac}\right) / 2 \mathrm{c}
$$

donde $x=\mathrm{EN}_{\mathrm{m}}(\mathrm{Mcal} / \mathrm{kg}), a=-0,41 \mathrm{EM}, b=$ $0,877 \mathrm{EM}+0,41 \mathrm{CMS}+\mathrm{EG}$, y $c=-0,877 \mathrm{CMS}$ (ZinnyShen, 1998). Donde, $\mathrm{EM}=0,056 \times \mathrm{W}^{0.75}, \mathrm{EG}=0,276$ $\times \mathrm{GDP} \times \mathrm{PV}^{0.75}$ y $\mathrm{CMS}=$ promedio de consumo de MS, kg/d (NRC, 1985; Estrada et al., 2013).

El comportamiento productivo (consumo de MS, GDP, eficiencia alimenticia) y la eficiencia en la utilización de la energía de la dieta (EN observada de la dieta, la relación EN observada/ esperada, y el consumo esperado de MS) se analizaron, utilizando el procedimiento MIXED de SAS (SAS Inst., version 9.3), como un diseño de bloques completos al azar considerado al peso inicial como criterio de bloqueo y a la corraleta como la unidad experimental. Los efectos del tratamiento fueron probados para componentes lineales, cuadráticas y cúbicas del nivel de suplementación ZEOL (SAS Inst., version 9.3). Los polinomios ortogonales se consideraron significativos cuando el valor de $p$ fue $\leq 0.05$, y se identificaron las tendencias cuando el valor de $\mathrm{p}$ fue $>0,05 \mathrm{y} \leq 0,10$. 


\section{RESULTADOS Y DISCUSIÓN}

Debido a que el efecto cúbico no fue significativo $(p>0,10)$ en ninguna de las variables evaluadas, se omitió el valor de significancia para ese componente.

De acuerdo con el análisis proporcionado por la empresa ZEOSIL (Grupo TCDN, Puebla, México) la zeolita utilizada en este experimento (depósito de San Juan Raya, región de Puebla; Puebla, México), estaba compuesta (g / kg de producto) de $\mathrm{SiO}_{2}(662), \mathrm{Al}_{2} \mathrm{O}_{3}$ (146), y CaO (27.3), concordando con la composición mineral de la zeolita natural (clinoptilolita-Ca) informado por EFSA (2013) y por Shadrikov y Petukhov (2014).

Los resultados del comportamiento productivo y de la eficiencia en la utilización de la EN de la dieta se muestran en el tabla II. La adición de zeolita no afectó el consumo de MS ( $p>0,73)$ ni la ganancia diaria ( $p>0,15)$; sin embargo, como resultado de un incremento numérico $(5,5 \%, p=0,15)$ en la ganancia diaria con el nivel de 3\% de ZEOL, se mejoró (componente cuadrático, $\mathrm{p}<0,01$ ) la eficiencia en la ganancia (ganancia/ consumo) con este tratamiento.

El comportamiento productivo observado para el grupo control (sin zeolita) concuerda con los informados en otros estudios en los cuales utilizaron raza, peso inicial y dietas similares a las utilizadas en este experimento. Ganancias diarias (rango de 0,230 a 0,291 $\mathrm{kg} / \mathrm{d}$ ), consumos de MS (rango de 0,230 a 0,291 kg/d) y eficiencias (rango de 0,180 a 0,210) similares a lo observado para el grupo control en este experimento se han informado para machos enteros cruzados de las razas Katahdin $\times$ Pelibuey consumiendo dietas de finalización libres de zeolita o de algún otro tipo de aditivo y con un contenido energético de 1,90 a 2,10 $\mathrm{Mcal} / \mathrm{kg}$ de EN para mantenimiento (López-Carlos et al., 2011; Ríos et al., 2010, 2014; Estrada et al., 2013, 2016). Por otra parte, la información sobre la respuesta productiva de borregos consumiendo dietas de finalización suplementadas con zeolitas es escasa. Aun así, estudios previos en ganado bovino han mostrado que la ingesta de alimento no se ve afectado por la inclusión de hasta 5\% de zeolita en dietas con bajo (Delgado et al., 1996) y moderado (Pulido y Fehring, 2004) contenido de energía. En cambio, en ovinos en finalización suplementados con zeolitas los resultados han sido inconsistentes. Por ejemplo, en un primer experimento (Pond, 1984) la adición de zeolita no afectó el consumo de MS de los corderos pero mejoró la ganancia diaria. Mientras que en un segundo experimento (Pond, 1989) la adición de $2 \%$ de zeolita a una dieta de finalización con $14 \%$ PC se reflejó en un incremento de consumo de MS acompañado de un aumento de la ganancia diaria de peso, mientras que con un nivel de $9 \%$ de PC en la dieta la suplementación con zeolita tuvo un efecto negativo sobre el comportamiento productivo.

Como resultado de la dilución energética de la dieta como consecuencia de la adición de zeolita, podría esperarse un ligero incremento en el consumo; sin embargo, por las propiedadesadsorbentes, que modifican la viscosidad de los fluidos, la zeolita (Spotti et al. 2005) podría disminuir la tasa de pasaje lo que se traduciría en un efecto compensatorio en la regulación

Tabla II. Influencia del nivel de suplementación de zeolita sobre el rendimiento productivo y utilización aparente de la energía neta de la dieta (Influence of zeolite supplementation level on growth performance and apparent dietary net energy).

\begin{tabular}{|c|c|c|c|c|c|c|c|}
\hline \multirow{2}{*}{ Concepto } & \multicolumn{4}{|c|}{ Zeolita en dieta, $\%$} & \multirow{2}{*}{ SEM } & \multicolumn{2}{|c|}{ Valor de $p$} \\
\hline & 0 & 1,5 & 3 & 4,5 & & L & $\mathrm{C}$ \\
\hline Réplicas & 5 & 5 & 5 & 5 & & & \\
\hline Días en prueba & 75 & 75 & 75 & 75 & & & \\
\hline \multicolumn{8}{|l|}{ Peso, kg } \\
\hline Inicial & 33,38 & 33,63 & 33,82 & 33,68 & 0,107 & 0,48 & 0,87 \\
\hline Final & 54,18 & 53,93 & 55,58 & 54,20 & 1,03 & 0,22 & 0,72 \\
\hline Consumo de MS, kg/d & 1,440 & 1,425 & 1,406 & 1,410 & 0,073 & 0,74 & 0,90 \\
\hline Ganancia diaria, kg/d & 0,277 & 0,271 & 0,290 & 0,274 & 0,013 & 0,74 & 0,15 \\
\hline Ganancia/consumo, kg/kg & $0,192^{a}$ & $0,190^{\mathrm{a}}$ & $0,206^{b}$ & $0,194^{a}$ & 0,008 & 0,84 & 0,01 \\
\hline \multicolumn{8}{|l|}{ EN de la dieta, Mcal/kg } \\
\hline Mantenimiento & $2,01^{a}$ & $2,00^{\mathrm{a}}$ & $2,13^{b}$ & $2,03^{a}$ & 0,020 & 0,16 & $<0,01$ \\
\hline Ganancia & $1,35^{\mathrm{a}}$ & $1,34^{a}$ & $1,46^{b}$ & $1,37^{\mathrm{a}}$ & 0,018 & 0,16 & $<0,01$ \\
\hline \multicolumn{8}{|l|}{ EN (observado/esperado) } \\
\hline Mantenimiento & $0,98^{a}$ & $0,99^{a}$ & $1,07^{b}$ & $1,03^{\mathrm{a}}$ & 0,012 & 0,16 & $<0,01$ \\
\hline Ganancia & $0,98^{\mathrm{a}}$ & $0,99^{a}$ & $1,07^{\mathrm{a}}$ & $1,03 a$ & 0,016 & 0,16 & $<0,01$ \\
\hline Consumo diario de MS (observado/esperado) & $1,02^{\mathrm{a}}$ & $1,01^{\mathrm{a}}$ & $0,93^{b}$ & $0,98^{a}$ & 0,015 & 0,16 & $<0,01$ \\
\hline
\end{tabular}

Superíndices diferentes en hileras indican diferencias $(p<0,05)$; $p=$ Nivel de significancia observado para el efecto lineal y cuadrático de nivel de suplementación de zeolita. 
del consumo. Como resultado de la característica inerte (no contiene materia que aporte energía) de la zeolita, por cada nivel de sustitución del maíz-pasta de soja se disminuyó la concentración de PC en 0,11 puntos porcentuales y la energía neta en $0,03 \mathrm{Mcal} / \mathrm{kg}$ (tabla I). Aun así, el rendimiento productivo no se vio afectado negativamente por la inclusión de zeolita. El hecho que la respuesta productiva de los ovinos no se afectara por el menor aporte de nutrientes en respuesta a cada incremento del nivel de zeolita puede estar relacionado con el uso más eficiente de algunos componentes de la dieta. Sadeghi y Shawrang (2006) reportaron una mayor digestión de las fracciones de fibra cuando $3 \%$ de zeolita fue incluida en la dieta. De la misma manera, Ruíz et al. (2008) observaron una mayor digestión de la fibra ácido detergente en el ganado bajo tratamiento con zeolita con respecto al grupo control. Lo anterior, pudo expresarse con una mayor eficiencia alimenticia (ganancia/consumo) cuando los niveles de zeolita fueron por encima de 1,5\% de la dieta. La forma más común de realizar evaluaciones de los efectos del tratamiento sobre la eficacia de la dieta se basan únicamente en la conversión alimenticia (consumo/ganancia) o en la eficiencia de ganancia (consumo/ganancia). Calculado a través de estas relaciones, la evaluación del valor alimenticio comparativo se confunde con el consumo de alimento y la ganancia. Basado en las medidas de rendimiento del crecimiento se puede predecir con bastante precisión (Cannas et al., 2004; NRC, 2007) la ingesta requerida de energía, y conociendo la composición de la dieta (el aporte teórico de energía), es posible predecir el consumo esperado de MS. La relación de consumo esperado vs observado revela diferencias en la eficiencia con independencia de la ganancia de peso, proporcionando información importante sobre los posibles efectos del tratamiento sobre la eficiencia de utilización de la energía de la dieta en sí, y son una aplicación importante y práctica que contemplan las normas actuales de la energética en la investigación nutricional (Zinn et al., 2008). De esta manera, cuando el coeficiente de EN observado-a-esperado es 1,00, significa que el comportamiento correspondió con el consumo de energía neta que teóricamente contenía la dieta, mientras que coeficientes por encima de 1,00 (v.gr. 1.04) indican que existió una mayor eficiencia en la utilización de la energíaneta que teóricamente contenía la dieta. Cuando se estima el coeficiente de consumo esperado vs consumo observado, la interpretación del coeficiente es exactamente lo opuesto. Valores por debajo de 1,00 significa mayor retención de energía por unidad de MS consumida (menor cantidad de unidades de MS requeridas para un rendimiento productivo dado). En ese sentido, el grupo control y la sustitución con 1,5\% de zeolita no modificaron el coeficiente de EN observada sobre la EN esperada de la dieta (coeficientes de 0,98 y 0,99 para inclusiones de 0 y 1,5\%, respectivamente),ni se modificó el coeficiente de unidades de MS requerida para ganancia (1,02 para inclusiones de 0 y 1,5\%; respectivamente, tabla II). Sin embargo, cuando el nivel de zeolita se incrementó por encima de 1,5\%, el coeficiente se aumentó en promedio en $5,5 \%$ y se redujo en $7,4 \%$ el coeficiente de unidades de MS requerida para ganancia; siendo el valor máximo (componente cuadrático, $\mathrm{p}<0,01$ ) para nivel de $3 \%$ de suplementación. En este experimento, el aumento del valor de la EN de la dieta y las reducción del coeficiente de consumo de MS esperado vs observado a altos niveles de zeolita mostró que la suplementación de zeolita a niveles mayores del 1,5\% mostró efectos positivos independientes de la composición química de la dieta. En experimentos previos en los cuales se utilizó la zeolita como aditivo alimenticio hasta en $4 \%$ en dietas altas en energía se detectaron cambios en la proporción de AGV en el rumen, incrementándose la proporción de propionato y disminuyéndose la proporción de acetato y de metano durante el proceso fermentativo (Galyean y Chabot, 1981; McCollum y Galyean, 1983). Más recientemente Urías-Estrada et al. (2016) demostraron una mejor utilización del almidón con una mayor proporciónde propionato con la disminución de la proporción acetato:propionato y una menor producción estimada de metano favoreciendo la eficiencia energética.

Como resultado de la afinidad de las zeolitas por los cationes de $\mathrm{NH}_{3}-\mathrm{N}$, en experimentos donde se ha probado la zeolita con resultados positivos en dietas de moderadas a bajas en energía (> 45\% de forraje en la dieta) se le ha atribuido respuesta positiva a una mejor retención del N. Datos in vivo e in vitro mostraron que hasta un 15 por ciento del $\mathrm{NH}_{4}^{+}$en el rumen puede ser absorbido por la zeolita (Mumpton y Fishman, 1977). Sin embargo, este efecto no ha sido significativo en dietas con alto contenido de energía (McCollum y Galyean, 1983; Urías-Estrada et al., 2015). Por lo tanto, para este tipo de dietas, el efecto de la zeolita sobre la mejora en la eficiencia energética de la dieta debe estar más relacionado con la mejora en tasas de fermentación ruminal y la utilización del almidón y de la materia orgánica.

\section{CONCLUSIONES}

El sustituir parcialmente la pasta de soja por zeolita hasta un nivel de 1,5\% en la dieta de ovinos de pelo no afectó negativamente el consumo de MS, la eficiencia alimenticia y la utilización aparente de la energía neta de la dieta. Al incrementar el nivel de suplementación por encima de $1,5 \%$ se mejoró la eficiencia alimenticia y la utilización aparente de la energía neta de la dieta, observándose un mayor efecto positivo con el nivel de inclusión de 3\%. Con respecto al grupo testigo, el $3 \%$ de inclusión de zeolita a la dieta mejoró en 6,8\% la eficiencia alimenticia y en $8,4 \%$ la utilización aparente de la energía neta de la dieta.

\section{BIBLIOGRAFÍA}

AOAC. 2000. Official Methods of Análisis. 17th edition. Association of Official Analytical Chemists. Gaithersburg, M.D. USA.

Cannas, A.; Tedeschi, L.O.; Fox, D.G.; Pell, A.N. and Van Soest, P.J. 2004. A mechanistic model for predicting the nutrient requirements and feed biological values for sheep. J Anim Sci, 82: 149-169.

Delgado, A.; Molina, A. and León, I. 1996. Zeolita como reguladora del consumo de proteína natural en añojos alimentados con forraje y suplementados con miel-urea. Rev Cubana Cs Agric, 30: 265.

EFSA. 2013. Scientific Opinion on the safety and efficacy of clinoptilolite of sedimentary origin for all animal species. European Food Safety Authority. EFSA Journal, 11: 3039-3052. 
Estrada-Angulo, A.; Valdés, Y.S.; Carrillo-Muro, O.; Castro-Pérez. B.I.; Barreras, A.; López-Soto, M.A.; Plascencia, A.; Dávila-Ramos, H.; Rios, F.G. and Zinn, R.A. 2013. Effects of feeding different levels of chromium-enriched live yeast in hairy lambs fed a corn-based diet: Effects on growth performance, dietary energetics, carcass traits and visceral organ mass. Anim Prod Sci, 53: 308-315.

Estrada-Angulo, A.; López-Soto, M.A.; Rivera-Méndez, C.R.; CastroPérez, B.I.; Ríos, F.G.; Dávila-Ramos, H.; Barreras, A.; Urías-Estrada, J.D.; Zinn, R.A. and Plascencia, A. 2016. Effects of combining feed grade urea and a slow-release urea product on performance, dietary energetics and carcass characteristics of feedlot lambs fed finishing diets with different starch to acid detergent fibre ratios. Asian-Australas J Anim Sci, 29: 1725-1733.

Galyean, M.L. and Chabot, R.C.1981 . Effect of sodium bentonite, buffer salts, cement kiln dust and clinoptilolite and rumen characteristics in beef steers fed a high roughage diet. J Anim Sci, 52: 1197-1204.

Ghaemnia, L.; Bojarpour, M.; Mirzadeh, K.H.; Chaji, M. and Eslami, M. 2010. Effect of different levels of zeolite on digestibility and some blood parameters in Arabic lambs. J Anim Vet Adv, 9: 779-781.

Goodarzi, M. and Nanekarani, S. 2012. The effects of calcic and potassicclinoptilolite on ruminal parameters in Lori breed sheep. APCBEE Procedia, 4: 140-145.

Inglezakis, V.J. and Zorpas, A. 2012. Natural zeolites structure and porosity. In: Handbook of natural zeolites. Inglezakis, V.J. and A.Zorpas (Eds.). Bentham Science Publisher. Sharjah UAE. pp. 133-146.

López-Carlos, M.A.; Ramírez, R.G.; Aguilera-Soto, J.I.; Aréchiga, C.F.; Plascencia, A.; Rincón, R.M.; Medina-Flores, C.A.; Rodríguez, H. and Gutiérrez-Bañuelos, H. 2011. Effect of duration of feeding zilpaterol or ractopamine on feedlot performance and carcass characteristics lambs. Livest Sci, 138: 251-258.

McCollum, F.T. and Galyean, M.L. 1983. Effects of clinoptilolite on rumen fermentation, digestion and feedlot performance in beef steers fed high concentrate diets. J Anim Sci, 56: 517-523.

Mumpton, F.A. 1999. La roca mágica: uses of natural zeolites in agriculture and industry. Proc Natl Acad Sci, 96: 3463-3470.

Mumpton, F.A. and Fishman, P.H. 1977. The application of natural zeolites in animal science and acquaculture. J Anim. Sci, 45: 1188-1203.

NOM-051-ZOO-1995. 1995. Trato humanitario en la movilización de animales (03.11.2015).

NOM-024-ZOO-1995. 1995. Estipulaciones de salud animal y características zoosanitarias durante el transporte de animales. http://198.61.233.93:8080/web/a_paginas/a_pdf/024_zoo. pdf (03.11.2015).

NOM-062-ZOO-1999. 1999. Especificaciones técnicas para la producción, cuidado y uso de los animales de laboratorio. http://www.fmvz. unam.mx/fmvz/principal/archivos/062ZOO.PDF (03.11.2015).

NRC. 1985. National Research Council. Nutrient Requirements of Domestic Animals. Nutrient Requirements of Sheep. Sixth Revised Edition, National Academy of Sciences, Washington, D.C. USA.

NRC. 2007. National Research Council. Nutrient requirements of small ruminants sheep, goats, cervids, and new world camelids. The National Academic Press. Washington, D.C. USA.

Ostrooumov, M. 2002. Zeolitas de México: Diversidad mineralógica y aplicaciones. Universidad Michoacana. San Nicolás de Hidalgo. http://mineralog.net/Articulos_electronicos/zeolitasMéxico.pdf (25.02.2016)

Pond, W.G. 1984. Response of growing lambs to clinoptilolite or zeolite NAA added to corn, corn-fish meal and corn-soybean meal diets. J Anim Sci, 59: 1320-1328.

Pond, W.G. 1989. Effects of dietary protein level and clinoptilolite on the weight gain and liver mineral response of growing lambs to copper supplementation. J Anim Sci, 67: 2772-2781.
Pulido, R.G. and Fehring, A. 2004. Efecto de la adición de una Zeolita natural sobre la respuesta productiva de terneras de lechería, postdestete. Arch Med Vet, 36: 197-201.

Ríos-Rincón, F.G.; Barreras-Serrano, A.; Estrada-Angulo, A.; Obregón, J.F.; Plascencia-Jorquera, A.; Portillo-Loera, J.J. and Zinn, R.A. 2010. Effect of level of dietary zilpaterol hydrochloride ( $\beta_{2}$-agonist) on performance, carcass characteristics and visceral organ mass in hairy lambs fed all-concentrate diets. J Appl Anim Res, 38: 33-38.

Ríos-Rincón, F.G.; Dávila-Ramos, H.; Estrada-Angulo, A.; Plascencia, A.; López-Soto, M.A.; Castro-Perez, B.I.; Calderón-Cortes, J. F.; PortilloLoera, J.J. and Robles-Estrada, J.C. 2014. Influence of protein and energy level on growth performance, dietary energetics and carcass characteristics of feedlot hair lambs. Asian-Australas J Anim Sci, 27: 55-60.

Ruíz, O.; Castillo, Y.; Elías, A.; Arzola, C.; Rodríguez, C.; Salinas, J.; La O, O. and Olguín, C. 2008. Efecto de cuatro niveles de zeolita en la digestibilidad y consumo de nutrientes en ovinos alimentados con heno de alfalfa y concentrado. Nota Técnica. Rev Cubana Cs Agric, 42: 367-370.

Sadeghi, A.A. and Shawrang, P. 2006. The effectof natural zeolite on nutrient digestibility, carcass traits and performance of Holstein steers given a diet containing urea. Anim Sci, 82: 163-167.

SAS. 2007. SAS/STAT: user's Guide: Statistics. Release 9.3. SAS Institute Inc. Cary, NC.

Shadrikov, A.S. and Petukhov, A.D. 2014. Natural zeolite-clinoptilolite characteristics determination and modification. National Tech University Ukrania. Technical Report. pp. 162-167 (UDC 544.02+546.05). Sherwood, D.M.; Erickson, G.E. and Klopfenstein, T.J. 2005. Effect of clinoptilolite zeolite on cattle performance and nitrogen volatilization loss. Nebraska Beef Cattle Reports. Paper 177.

Spotti, M.; Fracchiola, M.L.; Arioli, F.; Canoni, F. and Pompa, G. 2005. Aflatoxin $B_{1}$ binding to sorbents in bovine ruminal fluid. Vet Res Commun, 29: 507-515.

Urías-Estrada, J.A.; López-Soto, M.A.; Barreras, A.; Aguilar-Hernández, J.A.; González-Vizcarra, V.M.; Estrada-Angulo, A.; Zinn, R.A.; Mendoza, G.D. and Plascencia, A. 2016. Influence of zeolite (clinoptilolite) supplementation on characteristics of digestion and ruminal fermentation of steers fed a steam-flaked corn-based finishing diet. Anim Prod Sci. http://www.publish.csiro.au/AN/justaccepted/AN16128.

Ural, D. A. 2014. La eficacia de la suplementación de clinoptilolita sobre la producción de leche y el recuento de células somáticas. Rev MVZ Córdoba, 19: 4442-4448.

Van Soest, P.J.; Robertson, J.B. and Lewis, B.A. 1991.Methods for dietary fiber, neutral detergent fiber, and non starch polysaccharides in relation to animal nutrition. J Dairy Sci, 74: 3583-3597.

Zinn, R.A. and Shen, Y. 1998. An evaluation of ruminally degradable intake protein and metabolizable amino acid requirements of feedlot calves. J Anim Sci, 76: 1280-1289.

Zinn, R.A.; Barreras, A.; Owens, F.N. and Plascencia, A. 2008. Performance by feedlot steers and heifers: $A D G$, mature weight, $D M l$ and dietary energetics. J Anim Sci, 86: 1-10. 